\title{
Ursolic Acid Promotes the Sensitization of rhTRAIL-resistant Triple-negative Breast Cancer
}

\author{
JASMINE M. MANOUCHEHRI ${ }^{1,2}$ and MICHAEL KALAFATIS ${ }^{1,2,3}$ \\ ${ }^{1}$ Department of Chemistry, Science and Research Center, Cleveland State University, Cleveland, OH, U.S.A.; \\ ${ }^{2}$ Center for Gene Regulation in Health and Disease (GRHD), Cleveland State University, Cleveland, OH, U.S.A.; \\ ${ }^{3}$ Department of Molecular Cardiology, Lerner Research Institute, \\ Cleveland Clinic Foundation Cleveland, OH, U.S.A.
}

\begin{abstract}
Background/Aim: Triple-negative breast cancer (TNBC) can be characterized as the deadliest breast cancer type considering the lack of efficacious therapeutics. Recombinant human tumor necrosis factor-related apoptosisinducing ligand (rhTRAIL) is an encouraging anti-cancer therapeutic with the capacity to induce apoptosis in cancer cells but there are TNBCs less susceptible to rhTRAIL. The aim of this study was to assess the potential of the natural product ursolic acid (UA) to sensitize of rhTRAIL-resistant TNBCs. Materials and Methods: In order to evaluate apoptosis induction in rhTRAIL and UA-treated TNBC BT20 and HCC1937 cells that are resistant to rhTRAIL, western blot analysis and Annexin V/PI assays were executed. Results: UA increased the expression of death receptors 4 and 5 and decreased the expression of $c-F L I P_{L}$ transcriptionally sensitizing rhTRAIL-resistant TNBC cells to apoptosis induced by rhTRAIL. Conclusion: UA is a possible potent sensitizer of rhTRAIL-resistant TNBCs to rhTRAIL-induced apoptosis.
\end{abstract}

The most severe type of breast carcinomas often resulting in a poor prognosis are triple-negative breast cancers (TNBCs) (1). This highlights the priority for the development and application of multi-targeted and molecular-oriented therapies that are more effective and safer in these troublesome TNBCs in comparison to traditional chemotherapy and radiation therapy. TNBCs are very aggressive and difficult to treat using common antibreast cancer therapies such as hormone-targeted therapies,

Correspondence to: Michael Kalafatis, Department of Chemistry, Cleveland State University, 2351 Euclid Avenue, Science and Research Center SR370, Cleveland, OH 44115, U.S.A. Tel: +1 2166872460, Fax: +1 2166879298, e-mail:m.kalafatis@csuohio.edu

Key Words: Apoptosis, breast cancer, c-FLIPL, death receptors, ursolic acid, rhTRAIL. because these breast cancers lack the expression of human epidermal growth factor receptor-2 (HER2), estrogen receptor (ER), and progesterone receptor (PR). Therefore, it is imperative to produce hormone-independent targeted therapies (1).

Recombinant human tumor necrosis factor (TNF)-related apoptosis-inducing ligand (rhTRAIL), the optimized form of the endogenous death ligand TRAIL, is a compelling, wellstudied anti-cancer therapeutic (1-5). rhTRAIL is not toxic to normal, non-transformed cells, but maintains its ability to induce apoptosis in cancer cells (1-5). rhTRAIL induces apoptosis via the extrinsic pathway by triggering trimerization of death receptors (DRs) 4 and 5 and facilitating the activation of apoptotic associated proteins caspase 8 , executioner caspases 3,6 , and 7 along with pivotal poly (ADP-ribose) polymerase or PARP cleavage (6). Nonetheless, certain studies have shown a variable response of the majority of breast cancer cells to rhTRAIL treatment due to up-regulation of anti-apoptotic proteins such as cFLIP $_{\mathrm{L}}$ and down-regulation of DRs. rhTRAIL phase I clinical trials have been carried out but were discontinued due to inadequate therapeutic effect (1). Consequently, researchers have focused on developing and implementing divergent methods with co-treatments to overcome resistance to apoptosis through ascertaining capacities of different therapeutic agents derived from "mother nature" to enhance apoptosis induced by rhTRAIL in cancers that are resistant to rhTRAIL (7-16).

In our previous studies, the ability of "mother nature"derived compounds Quercetin (Q) and silibinin to sensitize cancers resistant to rhTRAIL to apoptosis induced by rhTRAIL has been demonstrated $(7,8,17)$. Q enhanced DR4 and DR5 expression in rhTRAIL-resistant malignant melanoma, and DR5 expression in rhTRAIL-resistant TNBC. The long form of c-FLIP (c-FLIP ${ }_{L}$ ) was degraded following treatment of rhTRAIL-resistant malignant melanoma and breast carcinoma with Q. Silibinin boosted expression of DR4 and DR5 and stimulated survivin down-regulation in 
rhTRAIL-resistant TNBCs. Thus, this evidence supports further studies that will examine other combinations of "mother nature"-derived compounds and rhTRAIL to treat rhTRAIL-resistant cancers.

One potential therapeutic agent of interest is ursolic acid (UA) that is extracted from the leaves and berries of various plants along with the coatings of fruits (18-20). UA can be consumed as a dietary supplement $(18,21)$. UA had an anti-cancer effect in breast, colon, prostate, hepatocellular, lung, and ovarian cancers (20-23). In vitro, UA stimulated apoptosis in breast, hepatocellular, and prostate cancers through the proper execution of both the extrinsic pathway and intrinsic pathway ending in PARP cleavage (characteristic hallmark of apoptosis) $(19,21,24$, 25). In prostate, gastric, and colorectal cancers UA induced apoptosis by increasing DR5 expression and decreasing survivin and Bcl-2 expression $(21,22,25,26)$. In vivo, UA decreased the growth of prostate and colorectal xenografts $(20,22)$. Previous studies have shown that UA does not impact non-tumorigenic, breast epithelial MCF-10A cell growth (27). Thus, UA warrants in-depth investigation as a potential sensitizer of TNBCs that are resistant to rhTRAIL.

Co-treatment with UA resulted in the sensitization of TNBC BT-20 and HCC1937 cells that are rhTRAILresistant. UA promoted the up-regulation of DRs along with c-FLIP ${ }_{\mathrm{L}}$ down-regulation facilitating the extrinsic pathway of apoptosis.

\section{Materials and Methods}

Drugs and chemicals. rhTRAIL was developed in accordance with earlier described methods (3-5). UA (molecular weight of $456.7 \mathrm{~g} / \mathrm{mol}$, Sigma, Milwaukee, WI, USA.) was dissolved in Polyethylene Glycol (PEG) molecular weight 400 (Fisher Scientific, Pittsburgh, PA, USA) at $10 \mathrm{mg} / \mathrm{ml}$.

Cell culture. Human breast cancer HCC1937 cells (ATCC CRL2336, Manassas, VA, USA), human breast cancer BT-20 cells (ATCC HTB-19, Manassas, VA, USA), and human non-tumorigenic breast epithelial MCF-10A cells (ATCC CRL-10317, Manassas, VA, USA) were cultured and treated with drugs for $72 \mathrm{~h}$ time periods and analyzed using the different assays described in this method section $(7,8)$.

Annexin V/PI assays-flow cytometry. Apoptosis was detected and examined as detailed in previous studies' protocols $(7,8)$.

Assessment of proteins associated with apoptosis. Cells were harvested and total cell lysates were prepared according to previously published protocols $(7,8)$. Western blot analysis was performed as described in previous studies' protocols using the primary antibodies: anti-PARP, anti-caspase 3, anti-cleaved caspase 3 , anti-caspase 8 , anti-cleaved caspase 8 , anti-DR4, and anti-DR5 (Cell Signaling Technology, Danvers, MD, USA), or anti-FLIP (Enzo Life Sciences, Farmingdale, NY, USA) $(7,8)$.
Flow cytometry analysis of DR4 and DR5 expression. Anti-DR4PE, anti-DR5-PE or isotype control mouse IgG1 kappa (eBioscience, Pittsburgh, PA, USA) were incubated with prepared cells in accordance to previously published protocols $(7,8)$. The isotype control is necessary for compensation of any non-specific binding. The expression of DR4 and DR5 was assessed on BD FACSCanto II flow cytometer.

Reverse Transcription-PCR analysis. Reverse transcription polymerase chain reaction (RT-PCR) was executed with primers for human DR5, human DR4, c-FLIP ${ }_{\mathrm{L}}$, and $\beta$-actin according to established protocols $(7,8)$.

Statistical analysis. Statistical analysis was performed using Student's $t$-test. $p$-Values less than 0.05 indicated statistically significant differences.

\section{Results}

Detecting rhTRAIL-induced apoptosis through FACS analysis. To determine UA's contribution to promoting apoptosis induced by rhTRAIL in TNBC cells, FACS analysis was carried out in TNBC cells treated for $72 \mathrm{~h}$ with 25 or $50 \mu \mathrm{M}$ UA in the presence or absence of $100 \mathrm{ng} / \mathrm{ml}$ rhTRAIL (Figure 1). rhTRAIL treatment $(100 \mathrm{ng} / \mathrm{ml})$ did not yield a considerable number of apoptotic TNBC cells relative to the vehicle-treated control $(p>0.05)$. UA potentiated rhTRAIL-induced apoptosis in TNBC BT-20 and HCC1937 cell lines. For instance, UA-treated $(50 \mu \mathrm{M})$ BT-20 and HCC 1937 cells yielded on average about $20 \%$ and $15 \%$ apoptotic cells, respectively $(p<0.05)$; meanwhile, the combined treatment of TNBC cells with $100 \mathrm{ng} / \mathrm{ml}$ rhTRAIL and $50 \mu \mathrm{M}$ UA produced on average about $30 \%$ apoptotic cells $(p<0.05)$.

Examining the execution of apoptosis. For the adequate apoptotic signal transduction, the execution of the caspase cascade is central, so the pathway of apoptosis was investigated by examining the expression levels of apoptotic associated proteins through western blot analysis. TNBC cells were treated with UA $(25$ or $50 \mu \mathrm{M})$ and rhTRAIL $(100 \mathrm{ng} / \mathrm{ml})$ or rhTRAIL alone for $72 \mathrm{~h}$. The levels of expression for various apoptotic proteins cleaved PARP, caspase 3 , and caspase 8 were ascertained; the combination treatments of UA and rhTRAIL augmented pro-apoptotic associated proteins (Figure 2). These findings affirm that the co-treatment of UA and rhTRAIL induced apoptosis by activating the extrinsic pathway as illustrated by PARP cleavage, caspase 8 activation, and caspase 3 activation. Moreover, it is noteworthy that PARP cleavage was not observed in TNBC cells treated with rhTRAIL in agreement with evidence obtained by other investigators that BT-20 and HCC1937 cells are rhTRAILresistant (28). Also, in a study, non-tumorigenic breast epithelial MCF-10A cell proliferation was not influenced by UA, and in another study by TRAIL, which further supports 

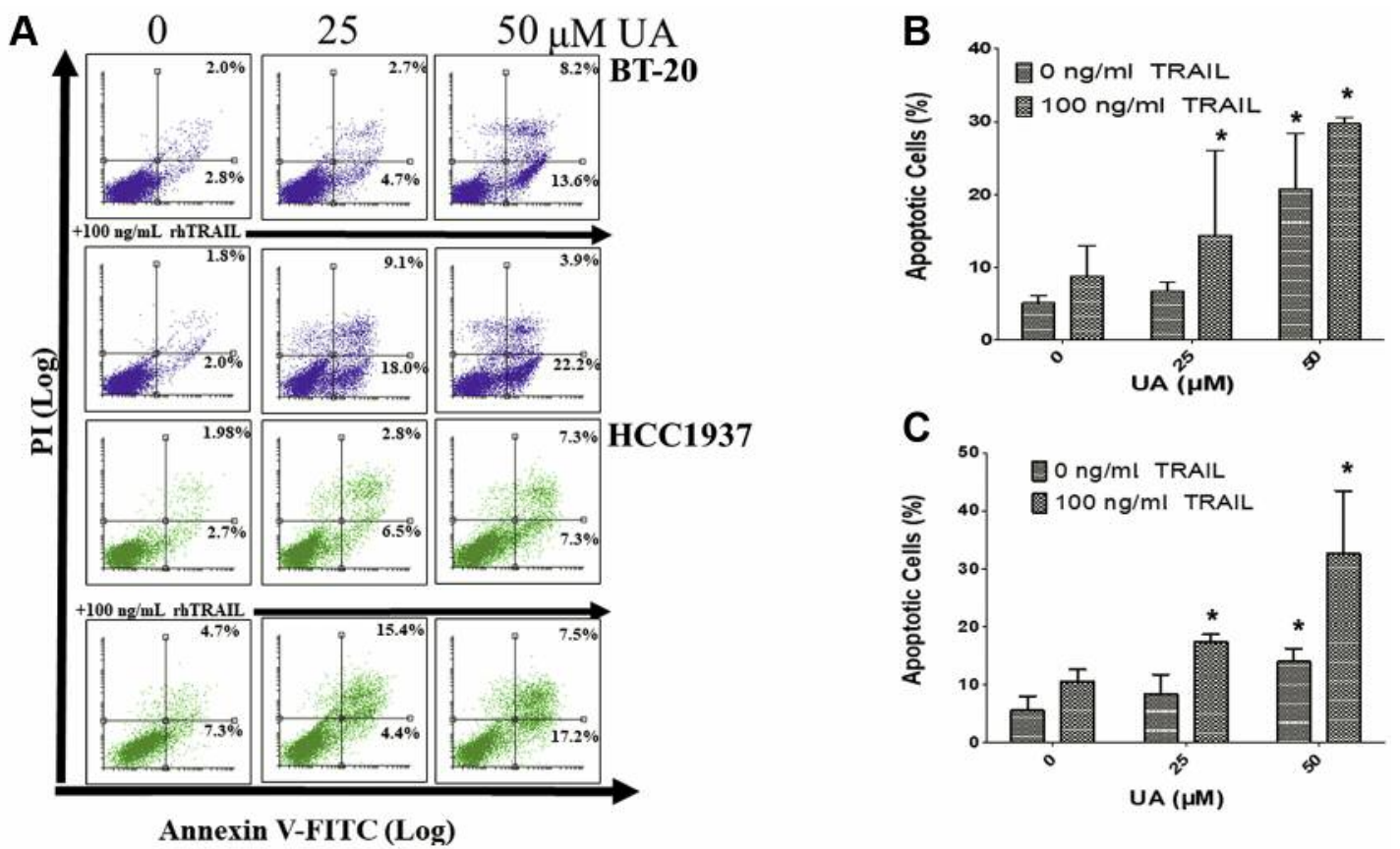

Figure 1. UA augmented apoptosis induced by rhTRAIL in TNBCs. TNBC cells treated with UA with and without $100 \mathrm{ng} / \mathrm{ml} \mathrm{rhTRAIL}$ over a $72-\mathrm{h}$ period. (A) The displayed plots obtained from FACS analysis, Annexin $V^{-} / P I^{+}$-dead cells are depicted in the left top quadrants, Annexin $V^{-} / P I^{-}$viable cells are depicted in the left bottom quadrants, Annexin $V^{+} / P I^{-}$-early apoptotic cells are depicted in the right bottom quadrants, and Annexin $V^{+} / P I^{+}$-late apoptotic, dead cells are depicted in the right top quadrants. Bar graphs (B) BT-20 and (C) HCC1937 illustrate the mean total percentage of apoptotic cells (Annexin $V^{+}$cells) \pm SEM derived from three independent experiments performed in triplicate $(n=9)$. * represents $p<0.05$.
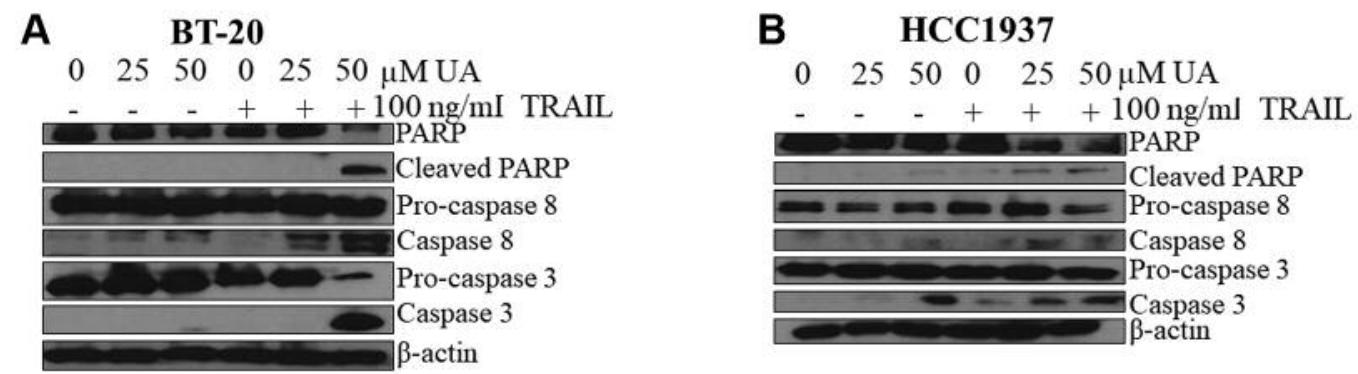

Figure 2. UA and rhTRAIL facilitate apoptosis by the extrinsic pathway. Co-treated TNBC (A) BT-20 and (B) HCC1937 cells were sensitized to rhTRAIL-induced apoptosis, as demonstrated by caspase 8 activation, caspase 3 activation, and PARP cleavage.

the proposal that the combinatorial therapeutic approach of UA and rhTRAIL influences only breast cancer cells and not normal, non-tumorigenic breast cells $(29,30)$. By taking the data compiled from western blot analysis (Figure 2) in association with the data compiled from FACS analyses (Figure 1), it is concluded that UA demonstrates the capability to intensify apoptosis induced by rhTRAIL in TNBCs that are rhTRAIL-resistant through the promotion of apoptosis via the extrinsic pathway.
UA's influence on the expression of DR4 and DR5. Flow cytometry and western blot analyses were carried out to identify UA's ability to impact protein and cell surface expression of DR4 and DR5 in TNBCs. The 72-h incubation period was selected following the completion of preliminary assays that determined UA's influence on the expression of DRs and c-FLIP $\mathrm{L}_{\mathrm{L}}$ occurred following $72 \mathrm{~h}$ incubation. UA significantly increased TNBC cells' DR5 protein expression in a dose-dependent manner $(p<0.05)$; also, UA significantly 
A
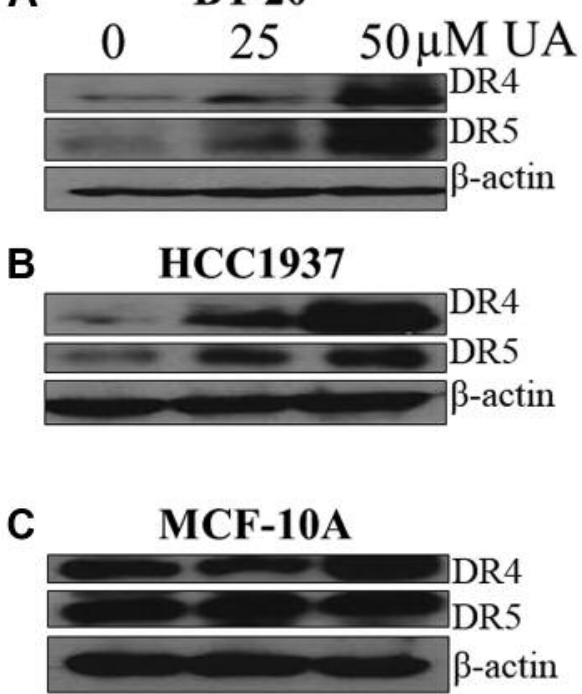
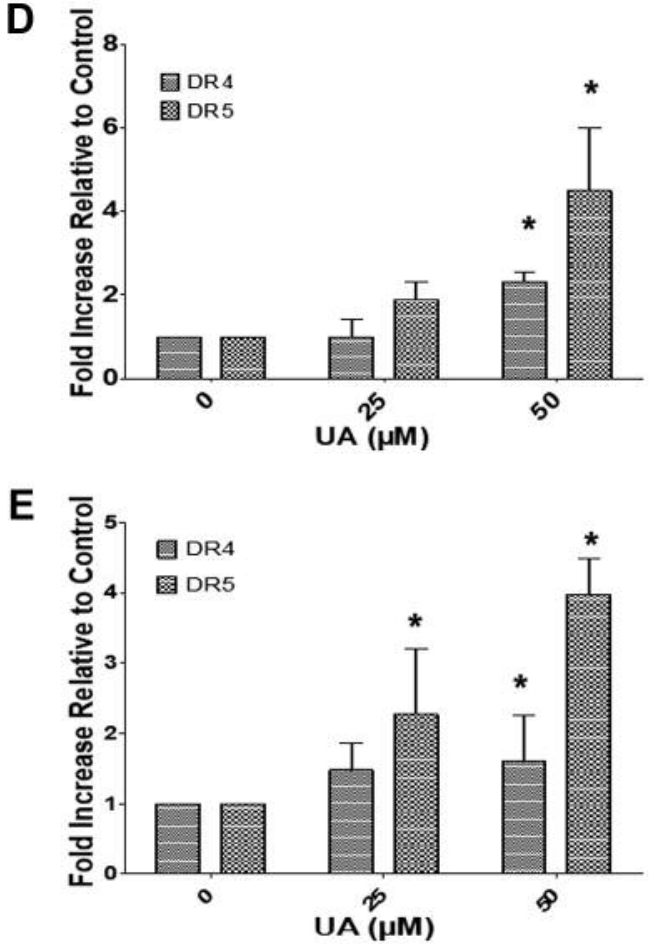

Figure 3. UA-treated cells' expression of DR4 and DR5. Protein levels of DR4 and DR5 (mature form) were determined in (A) BT-20 cells and (B) HCC1937; UA boosted DR4 and DR5 expression in TNBCs following $72 \mathrm{~h}$ of treatment in a dose-dependent manner. (C) Protein levels of DR4 and DR5 (mature form) were examined in UA-treated MCF-10A cells, and expression of DRs was not changed. Flow cytometry analysis of cell surface expression of DR4 and DR5 in (D) BT-20 and (E) HCC1937 cells treated with UA; the bar graphs illustrate the mean fold increase in the cell surface expressions of DR4 or DR5 relative to the vehicle-treated control \pm SEM from three independent experiments performed in triplicate $(n=9)$. * represents $p<0.05$.

increased DR4 protein expression in TNBC cells $(p<0.05)$ (Figures 3A and 3B). Cell surface expressions of DR4 and DR5 in UA -treated TNBC cells were assessed via FACS analyses (Figure 3D and E) and were in agreement with the heightened TNBC cell protein expression of DRs. The expression of DRs were not changed in MCF-10A cells following incubation with UA for $72 \mathrm{~h}$, (Figure 3C). UA stimulated DR4 and DR5 up-regulation in TNBC cells; the enhanced DRs' expression further verifies the role of UA in increasing the sensitiveness of TNBCs to rhTRAIL.

c-FLIP ${ }_{L}$ expression change due to UA. On the basis of the results demonstrating UA's capability to assist in elevating expression of caspase 8 , UA's effect on c-FLIP (caspase 8 inhibitor) expression was investigated. Findings obtained from western blot analysis (Figure 4A) establish that UA dose-dependently diminished the expression of c-FLIP $_{\mathrm{L}}$ in TNBC cells after $72 \mathrm{~h}$ of incubation.

$R T-P C R$ analyses of DRs and $c-F L I P_{L}$. RT-PCR analyses were performed with $\beta$-actin as a positive control to examine the potential transcriptional effects of UA on DR4, DR5, c-
FLIP $_{\mathrm{L}}$. The results show that DR4 and DR5 mRNA expressions were increased in UA-treated TNBC cells (Figure 4B). In addition, c-FLIP ${ }_{\mathrm{L}}$ mRNA expression was decreased transcriptionally in TNBC cells. Thus, UA's molecular mechanism for DR4 and DR5 up-regulation along with c-FLIP $\mathrm{L}_{\mathrm{L}}$ down-regulation is transcriptionally controlled.

\section{Discussion}

One of the commonly diagnosed cancers in women is breast cancer, so it is essential that multi-targeted and effective therapies are developed to deter a poor prognosis especially in TNBCs. A preferred mechanism of therapies for breast cancers is the induction of apoptosis. The conventional treatment methods of chemotherapy and radiation are administered to induce apoptosis and treat breast cancer, but the long-term application of these treatments usually worsen patients' conditions. Chemotherapy and radiation require a functional, non-mutated TP53 gene for the absolute activation of apoptosis through the intrinsic pathway. On the contrary, rhTRAIL does not need a functional p53 gene to activate the extrinsic pathway of apoptosis. Therefore, rhTRAIL is of 
A

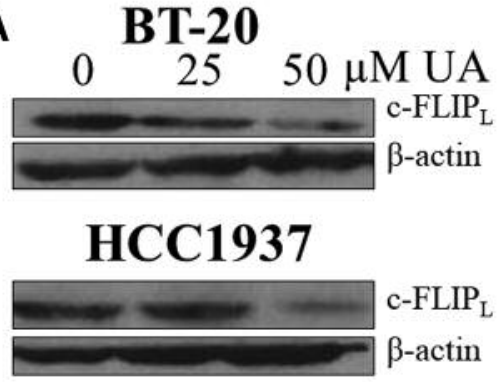

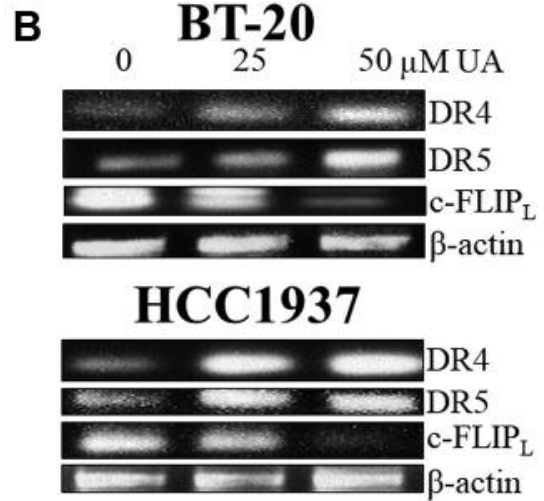

Figure 4. UA transcriptionally controls c-FLIP ${ }_{L}$ down-regulation and DRs up-regulation. (A) Western blot analysis showing that UA decreased the expression of c-FLIP ${ }_{L}$ in TNBC cells in a dose-dependent manner. (B) RT-PCR was executed with $\beta$-actin as a positive control in UA-treated (72 h) BT-20 and HCC1937 cells. UA diminished c-FLIP ${ }_{L}$ mRNA expression in TNBC cells and boosted DR4 and DR5 mRNA expression in TNBC cells.

interest as an anti-cancer therapy over traditional chemotherapy and radiation because there are many cancers that possess a mutated or nonfunctional p53 gene hindering this therapeutic strategy's effectiveness and can often result in worsening the condition of patients due to negative side effects $(1-4,31,32)$. Investigations have focused on applying additional compounds, including "mother-nature"-derived compounds, to enhance rhTRAIL's pro-apoptotic effects in an array of cancer cells including breast cancer cells (7-16, 33$35)$. Our laboratory has specifically examined the impact of quercetin in breast cancer and malignant melanoma and silibinin in breast cancer to sensitize cancer cells resistant to rhTRAIL $(7,8,17)$. The natural compound UA is a triterpenoid, and it should be noted that other laboratories have studied the positive impact of synthetic triterpenoids in combination with TRAIL on cancer cells in vitro and in vivo (33-35). Accordingly, this study's main objective was to apply rhTRAIL in conjunction with the natural compound UA as a strategy to treat TNBCs.

In order to appraise the TNBC cells' reactions to the cotreatment of UA and rhTRAIL, apoptosis detection assays and western blot analysis were carried out. Noteworthy, the effect of UA and rhTRAIL has not been examined in TNBC cells. Data were collected from both assessments, and it was deduced that UA intensified apoptosis induced by rhTRAIL in TNBC cells by activating the extrinsic pathway as illustrated by increased PARP cleavage, caspase 8 activation, and executioner caspase 3 activation. Based on these findings, UA's sensitization mechanism had to be elucidated.

Some researchers have hypothesized that there is a deficiency of DR4 and DR5 expression preventing the extrinsic pathway of apoptosis being executed fully via rhTRAIL treatment $(36,37)$. Hence, through western blot and FACS analyses on UA-treated TNBC cells, DR4 and
DR5 protein cellular and cell surface expression levels were investigated. The culminated data revealed that UA prompted increased expression of DR4 and DR5 in BT-20 and HCC1937 cells. UA treatment did not affect the expression of DRs in MCF-10A cells. Thus, UA's ability to up-regulate the expression of DRs is recognized in breast cancer cells but not in normal, non-transformed breast cells.

Following this outcome, the underlying molecular mechanism for UA's sensitizing effects was further examined through RT-PCR analysis. DR4 and DR5 were transcriptionally up-regulated by UA, and c-FLIP $\mathrm{L}_{\mathrm{L}}$ was transcriptionally down-regulated.

UA sensitizes TNBC cells to treatment with rhTRAIL by inducing expression of DRs and diminishing expression of c-FLIP $\mathrm{L}_{\mathrm{L}}$ and thus promoting apoptosis. Therefore, UA is a compelling sensitizer for TNBCs that are resistant to rhTRAIL and strongly justifies more investigation in vivo.

\section{Conflicts of Interest}

The Authors have no conflicts of interest to disclose regarding this study.

\section{Acknowledgements}

This work was supported in part by a grant from the Parker Hannifin Foundation.

\section{References}

1 Kuijlen J, Bremer E, Mooij J, Dunnen D and Helfrich W: Review: On TRAIL for malignant glioma therapy? Neuropath Applied Neurobiol 36: 168-182, 2010.

2 Wang D and Shi L: High-level expression, purification, and in vitro refolding of soluble tumor necrosis factor-related apoptosisinducing ligand (TRAIL). Applied Biochem and Biotech 157: 19, 2009. 
3 Shen YL, Zhang Y, Sun AY, Xia XX, Wei DZ and Yang SL: High-level production of soluble tumor necrosis factor-related apoptosis-inducing ligand (Apo2L/TRAIL) in high-density cultivation of recombinant Escherichia coli using a combined feeding strategy. Biotech Letters 26: 981-984, 2004.

4 Luo Q, Shen YL, Wei DZ and Cao W: Optimization of culture on the overproduction of TRAIL in high-cell-density culture by recombinant Escherichia coli. Applied Microbio Biotech 71: 184-191, 2006.

5 Turner KA, Lindner D and Kalafatis M: Recombinant human tumor necrosis factor-related apoptosis-inducing ligand selectively induces apoptosis in malignant melanoma. Int $\mathbf{J}$ Cancer Oncol 4: 1-8, 2017.

6 Fulda S and Debatin KM: Extrinsic versus intrinsic apoptosis pathways in anticancer chemotherapy. Oncogene 25: 4798-4811, 2006.

7 Manouchehri JM and Kalafatis M: Sensitization of rhTRAILresistant triple-negative breast carcinoma through silibinin cotreatment. Anticancer Res 37: 6593-6599, 2017.

8 Manouchehri JM, Turner KA and Kalafatis M: TRAIL-induced apoptosis in TRAIL-resistant breast carcinoma through Quercetin cotreatment. Breast Cancer: Basic and Clinical Res 12: 1-12, 2018.

9 Reinhardt A, Liu H, Ma Y, Zhou Y, Habbel JP, Possinger K and Eucker J: Tumor cell-selective synergism of TRAIL- and ATRAinduced cytotoxicity in breast cancer cells. Anticancer Res 38: 52669-52682, 2018.

$10 \mathrm{Kim}$ EY and Kim AK: Combination effect of Equol and TRAIL against human cervical cancer cells. Anticancer Res 33: 903-912, 2013.

11 Abdelhamed S, Yokoyama S, Refaat A, Ogura K, Yagita H, Awale S and Saiki I: Piperine enhances the efficacy of TRAILbased therapy for triple-negative breast cancer cells. Anticancer Res 34: 1893-1899, 2014.

12 Lee SC, Cheong HJ, Kim SJ, Yoon J, Kim JH, Kim KH, Kim SH, Kim HJ, Bae SB, Kim CK, Lee N, Lee KT, Park SK, Hong DS, Park HS and Won JH: Low-dose combinations of LBH589 and TRAIL can overcome TRAIL-resistance in colon cancer cell lines. Anticancer Res 31: 3385-3394, 2011.

13 Pawlak A, Miguel DD, Kutkowska J, Obminska-Mrukowicz B, Rapak A, Martinez-Lostao L: Flavopiridol strongly sensitizes canine lymphoma cells to TRAIL-induced apoptosis. Anticancer Res 37: 6655-6665, 2017.

14 Park S, Shim SM, Nam SH, Andera L, Suh N and Kim I: CGP74514A enhances TRAIL-induced apoptosis in breast cancer cells by reducing X-linked inhibitor of apoptosis protein. Anticancer Res 34: 73557-3562, 2014.

15 Bousserouel S, Bour G, Kauntzi H, Gosse F, Marescaux J and Paul F: Silibinin inhibits tumor growth in a murine orthotopic hepatocarcinoma model and activates the TRAIL apoptotic signaling pathway. Anticancer Res 32: 72455-2462, 2012.

16 Choi WY, Jin CY, Han MH, Kim GY, Kim ND, Lee WH, Kim SK and Choi YH: Sanguinarine sensitizes human gastric adenocarcinoma AGS cells to TRAIL-mediated apoptosis via down-regulation of AKT and activation of caspase-3. Anticancer Res 29: 4457-4465, 2009.

17 Turner K, Manouchehri $\mathbf{J}$ and Kalafatis M: Sensitization of recombinant human tumor necrosis factor-related apoptosisinducing ligand-resistant malignant melanomas by quercetin. Melanoma Res 28: 277-285, 2018.
18 Ikeda Y, Murakami A and Ohigashi H: Ursolic acid: An anti- and pro-inflammatory triterpenoid. Mol Nutrition \& Food Res 52: 26-42, 2008.

19 Weng H, Tan ZJ, Hu YP and Shu YJ: Ursolic acid induces cell cycle arrest and apoptosis of gallbladder carcinoma cells. Cancer cell Int 14: 1-10, 2014.

20 Shanmugam M, Rajendran P, Li F, Nema T, Vali S, Abbasi T, Kapoor S, Sharma A, Kumar A, Ho P, Hui K and Sethi G: Ursolic acid inhibits multiple cell survival pathways leading to suppression of growth of prostate cancer xenograft in nude mice. J Mol Med 89: 713-727, 2011.

21 Shanmugam M, Dai X, Kumar A, Tan B, Sethi G and Bishayee A: Ursolic acid in cancer prevention and treatment: Molecular targets, pharmacokinetics and clinical studies. Biochem Pharmacol 85: 1579-1587, 2013.

22 Prasad S, Yadav V, Sung B, Reuter S, Kannappan R, Deorukhkar A, Diagaradjane P, Wei C, Baladandayuthapani V, Krishnan S, Guha $S$ and Aggarwal B: Ursolic acid inhibits growth and metastasis of human colorectal cancer in an orthotopic nude mouse model by targeting multiple cell signaling pathways: Chemosensitization with capecitabine. Clin Cancer Res 18: 4942-4953, 2012.

23 Zhang Y, Kong C, Zeng Y, Wang L, Li Z, Wang H, Xu C and Sun Y: Ursolic acid induces PC3 cell apoptosis via activation of JNK and inhibition of Akt pathways in vitro. Mol Carcinogen 49: 374-385, 2010.

$24 \mathrm{Kim} \mathrm{K}$, Seo H, Choi H, Choi I, Shin Y and Ko SG: Induction of apoptotic cell death by ursolic acid through mitochondrial death pathway and extrinsic death receptor pathway in MDA-MB-231 cells. Arch Pharm Res 34: 1363-1372, 2011.

25 Kassi E, Papoutsi Z, Pratsinis H, Aligiannis N, Manoussakis M and Moutsatsou P: Ursolic acid, a naturally occurring triterpenoid, demonstrates anticancer activity on human prostate cancer cells. J Cancer Res Clin 133: 493-500, 2007.

26 Wang X, Zhang F, Yang L, Mei Y, Long H, Zhang X, Zhang J, Qimuge-Suyila QS and Su X: Ursolic acid inhibits proliferation and induces apoptosis of cancer cells in vitro and in vivo. $\mathrm{J}$ Biomed Biotechnol 2011: 1-8, 2011.

27 Mishra T, Arya R, Meena S, Joshi P, Pal M, Meena B, Upreti, Rana and Datta D: Isolation, characterization and anticancer potential of cytotoxic triterpenes from Betula utilis Bark. PLOS One 11: 1-14, 2016.

28 Rahman M, Pumphrey JG and Lipkowitz S: The TRAIL to targeted therapy of breast cancer. Adv. Cancer Res 103: 43-73, 2009.

29 Jahanafrooz Z, Motameh N and Bakhshandeh B: Comparative evaluation of silibinin effects on cell cycling and apoptosis in human breast cancer MCF-7 and T47D cell lines. Asian Pac J Cancer Prev 17: 2661-2665, 2016.

30 Dong Y, Yin S, Li J, Jiang C, Ye M and Hu H: Bufadienolide compounds sensitize human breast cancer cells to TRAILinduced apoptosis via inhibition of STAT3/Mcl-1 pathway. Apoptosis 16: 394-403, 2011.

31 Liu J, Zhang C, Hu W and Feng Z: Tumor suppressor p53 and its mutants in cancer metabolism. Cancer Letters 356: 197-203, 2015.

32 Guan YS, He Q and Zou Q: Status quo of p53 in the treatment of tumors. Anti-Cancer Drugs 27: 811-818, 2016.

33 Hyer ML, Croxton R, Krajewska M, Krajewski S, Kress CL, Lu M, Suh N, Sporn MB, Cryns VL, Zapata JM and Reed JC: 
Synthetic triterpenoids cooperate with tumor necrosis factorrelated apoptosis-inducing ligand to induce apoptosis in breast cancer cells. Cancer Res 65: 4799-4808, 2005.

34 Hyer ML, Ranxin S, Krajewska M, Myer C, Lebedeva IV, Fisher PB and Reed JC: Apoptotic activity and mechanism of 2-cyano3,12-dioxoolean-1,9-dien-28-oic-acid and related synthetic triterpenoids in prostate cancer. Cancer Res 68: 2927-2933, 2008.

35 Liby K, Royce DB, Williams CR, Risingsong R, Yore MM, Honda T, Gribble GW, Dmitrovsky E, Sporn TA and Sporn MB: The synthetic triterpenoids CDDO-methyl ester and CDDO-ethyl amide prevent lung cancer induced by vinyl carbamate. Cancer Res 67: 2414-2419, 2007.
36 Jung YH, Heo J, Lee Y, Kwon T and Kim YH: Quercetin enhances TRAIL-induced apoptosis in prostate cancer cells via increased protein stability of death receptor 5 . Life Sciences 86 : 351-357, 2010.

37 Kim J, Kim E, Park S, Lim J, Kwon T and Choi K: Quercetin sensitizes human hepatoma cells to TRAIL-induced apoptosis via Sp1-mediated DR5 up-regulation and proteasome-mediated cFLIPS down-regulation. J Cell Biochem 105: 1386-1398, 2008.

Received August 15, 2018

Revised November 1, 2018

Accepted November 2, 2018 